JOURNAL OF THE AMERICAN MATHEMATICAL SOCIETY

Volume 10, Number 1, January 1997, Pages 243-258

S 0894-0347(97)00223-3

\title{
SINGULARITIES OF THETA DIVISORS AND THE BIRATIONAL GEOMETRY OF IRREGULAR VARIETIES
}

\author{
LAWRENCE EIN AND ROBERT LAZARSFELD
}

\section{INTRODUCTION}

The purpose of this paper is to show how the generic vanishing theorems of [GL1] and [GL2] can be used to settle a number of questions and conjectures raised in [Kol3], Chapter 17, concerning the geometry of irregular complex projective varieties. Specifically, we focus on three sorts of results. First, we establish a well known conjecture characterizing principally polarized abelian varieties whose theta divisors are singular in codimension one. Secondly, we study the holomorphic Euler characteristic of varieties of general type having maximal Albanese dimension: we verify a conjecture of Kollár for subvarieties of abelian varieties, but show that it fails in general. Finally, we give a surprisingly simple new proof of a fundamental theorem of Kawamata $[\mathrm{Ka}]$ on the Albanese mapping of varieties of Kodaira dimension zero.

Turning to a more detailed description, we start with the singularities of theta divisors. Let $A$ be an abelian variety of dimension $g \geq 2$, and let $\Theta \subset A$ be a principal polarization on $A$, i.e. an ample divisor such that $h^{0}\left(A, \mathcal{O}_{A}(\Theta)\right)=1$. Ever since the work $[\mathrm{AM}]$ of Andreotti and Mayer on the Schottky problem, there has been interest in understanding what sort of singularities $\Theta$ can have. A wellknown theorem of Kempf $[\mathrm{K}]$ states that if $A$ is a Jacobian, then $\Theta$ has only rational singularities. For an arbitrary principally polarized abelian variety $(A, \Theta)$, Arbarello and DeConcini $[\mathrm{AD}]$ conjectured that if $\operatorname{dim} \operatorname{Sing}(\Theta)=g-2$ then $(A, \Theta)$ splits as a non-trivial product, i.e. that there exist principally polarized abelian varieties $\left(A_{1}, \Theta_{1}\right)$ and $\left(A_{2}, \Theta_{2}\right)$ such that

$$
A=A_{1} \times A_{2} \text { and } \Theta=p r_{1}^{*} \Theta_{1}+p r_{2}^{*} \Theta_{2} .
$$

Kollár [Kol3, Theorem 17.13] recently put these matters into a new perspective by proving that the pair $(A, \Theta)$ is $\log$ canonical. Denoting by $\Sigma_{k}(\Theta)$ the multiplicity locus

$$
\Sigma_{k}(\Theta)=\left\{x \in A \mid \operatorname{mult}_{x}(\Theta) \geq k\right\}
$$

Received by the editors March 29, 1996 and, in revised form, June 20, 1996

1991 Mathematics Subject Classification. Primary 14K25, 14J99, 32J27.

Key words and phrases. Singularities, theta divisors, generic vanishing theorem, Albanese mapping.

The first author was partially supported by N.S.F. Grant DMS 93-02512.

The second author was partially supported by N.S.F. Grant DMS 94-00815.

(C)1997 American Mathematical Society 
Kollár's theorem implies in particular that

$$
\text { every component of } \Sigma_{k}(\Theta) \text { has codimension } \geq k \text { in } A \text {. }
$$

For example, taking $k=g+1$ it follows that $\Theta$ can have no points of multiplicity $>g$. Using a very pleasant enumerative argument, Smith and Varley [SV] subsequently established that if $\Theta$ contains a $g$-fold point, then $(A, \Theta)$ splits as a product of $g$ elliptic curves (see $[\mathrm{Nak}]$ for a somewhat different approach).

Our first result shows that the conclusion of Kempf's theorem holds quite generally, and that in fact any example on the boundary of Kollár's theorem is split:

Theorem 1. If $\Theta \subset A$ is an irreducible theta divisor, then $\Theta$ is normal and has only rational singularities.

Corollary 2. If $(A, \Theta)$ is any principally polarized abelian variety, and if $k \geq 2$, then $\Sigma_{k}(\Theta)$ contains an irreducible component of codimension $k$ in $A$ if and only if $(A, \Theta)$ splits as a $k$-fold product of p.p.a.v.'s.

When $k=g$ we recover the theorem of Smith and Varley; the case $k=2$ gives the conjecture of Arbarello and DeConcini.

The proof of Theorem 1 is surprisingly quick. In brief, let $X \longrightarrow \Theta$ be a resolution of singularities. By applying the generic vanishing theorems on $X$, and arguing with some Nadel-type adjoint ideals on $A$, one reduces to showing that $\chi\left(X, \omega_{X}\right)>0$. But $X$ is of general type, and the inequality in question emerges as a special case of a conjecture of Kollár, which we discuss next.

Consider then a smooth projective variety $X$ of dimension $n$, and assume that the Albanese mapping

$$
\operatorname{alb}_{X}: X \longrightarrow \operatorname{Alb}(X)
$$

is generically finite, or in other words that $X$ has maximal Albanese dimension. A result of [GL1] asserts that under these circumstances $\chi\left(X, \omega_{X}\right) \geq 0$. If $X$ is birationally the product of a torus and some other variety then of course $\chi\left(X, \omega_{X}\right)=0$. But Kollár conjectured [Kol2, (17.9)] that if $X$ is of general type, then $\chi\left(X, \omega_{X}\right)>$ 0 . Our second result shows that this is true if $X$ is birationally a subvariety of $\operatorname{Alb}(X)$ :

Theorem 3. ${ }^{1}$ Let $X$ be a smooth projective variety of maximal Albanese dimension, and suppose that $\chi\left(X, \omega_{X}\right)=0$. Then the Albanese image

$$
\operatorname{alb}_{X}(X) \subseteq \operatorname{Alb}(X)
$$

of $X$ is fibred by tori. In particular, if $\operatorname{alb}_{X}: X \longrightarrow \operatorname{Alb}(X)$ is birational onto its image then $X$ is not of general type.

This is more than enough to give the inequality required for Theorem $1 .{ }^{2}$ For smooth subvarieties of abelian varieties, an equivalent statement was established independently by Qi Zhang [Z]. We complete the picture by showing that Kollár's conjecture fails in general. Our example is a threefold whose Albanese mapping is a branched covering with a rather degenerate branch divisor.

\footnotetext{
${ }^{1}$ This proof of this result builds on discussions some years ago with M. Green, and it should be considered at least partially joint work with him.

${ }^{2}$ In the interests of truth in advertising, we note that for Theorem 1 one only needs Kollár's conjecture when $X$ is birational to an irreducible theta divisor, and this case is covered by an old result of Kawamata and Viehweg [KV]. So in fact Theorem 1 can be read independently of the rest of the paper. However we naturally prefer to see it as part of a broader picture.
} 
Finally, we turn to varieties of Kodaira dimension zero. Let $X$ be a smooth projective variety of dimension $n$. Kawamata [Ka] showed that if $\kappa(X)=0$, then the Albanese mapping $\operatorname{alb}_{X}: X \longrightarrow \operatorname{Alb}(X)$ is surjective. By a standard covering argument, it is enough to prove this assuming that $P_{1}(X) \neq 0$, where as usual $P_{m}(X)=h^{0}\left(X, \omega_{X}^{\otimes m}\right)$ denotes the $m^{\text {th }}$ plurigenus of $X$. Kawamata's result is therefore a consequence of

Theorem 4. If $P_{1}(X)=P_{2}(X)=1$, then the Albanese mapping of $X$ is surjective.

Several other effective versions of Kawamata's theorem were previously given by Kollár ([Kol1], [Mori], [Kol2], [Kol3]), the strongest of which states that alb ${ }_{X}$ is surjective as soon as $P_{3}(X)=1$. Kollár also asked for analogous results involving $P_{2}$.

However the main interest of Theorem 4 derives not so much from any numerical improvements as from the the surprising simplicity and transparency of its proof. Kawamata's approach involved some rather difficult positivity results for direct images of dualizing sheaves, which were gradually replaced in Kollár's work by subtle arguments with vanishing theorems. By contrast, granting the general results of [GL2], Theorem 4 requires only a few lines. A pleasant geometric argument also recovers the more precise statement from [Ka] that if $\kappa(X)=0$, then the fibres of $\operatorname{alb}_{X}: X \longrightarrow \operatorname{Alb}(X)$ are connected, as well as one of Kollár's characterizations of abelian varieties. We hope that some of these ideas may find other applications in the future.

The paper is organized as follows. In section 1 we review for the convenience of the reader the results we use from [GL1] and [GL2], and we prove Theorem 3 . The applications to varieties of Kodaira dimension zero occupy $\S 2$, while $\S 3$ is devoted to theta divisors. We also include in $\S 3$ an extension of Kollár's theorem to singularities of pluri-theta divisors, as proposed in [Kol3], Problem 17.15. We reiterate that $\S 3$ may be read independently of the rest of the paper.

We have profitted from discussions with E. Arbarello, R. Donagi, M. Green, J. Kollár, R. Smith, R. Varley and J. Wahl. In particular, the statement of Theorem 1 was suggested by Kollár and Wahl, and as noted above discussions with Green played a substantial role in the proof of Theorem 3.

\section{Notation AND CONVENTIONS}

(0.1). We work throughout over the complex numbers $\mathbf{C}$.

(0.2). Given a smooth variety or complex manifold $X$ of dimension $n$, we generally denote by $\omega_{X}=\Omega_{X}^{n}$ the canonical line bundle of $X$. An exception occurs in our discussion of adjoint ideals at the beginning of $\S 3$, where in accordance with the standard notation of higher dimensional geometry, we use $K_{X}$ to denote (the linear equivalence class of) a canonical divisor on $X$.

(0.3). If $D$ and $E$ are divisors on a variety or complex manifold $X$, we write $D \prec E$ to indicate that $E-D$ is effective.

\section{Positivity of holomorphic Euler characteristics}

We start by recalling the material from [GL1] and [GL2] that will be needed here and in $\S 2$. Then we give the proof of Theorem 3 and the counter-examples to the general case of Kollár's conjecture. 
Review of generic vanishing theorems. Let $X$ be a compact Kähler manifold of dimension $n$, and as usual let $\operatorname{Pic}^{0}(X)$ be the complex torus parametrizing topologically trivial line bundles on $X$. Given a point $y \in \operatorname{Pic}^{0}(X)$, we denote by $P_{y}$ the corresponding topologically trivial bundle on $X$. For $0 \leq i \leq n$ consider the analytic subvarieties of $V_{i}(X) \subset \operatorname{Pic}^{0}(X)$ defined by:

$$
\begin{aligned}
V_{i}(X) & =\left\{y \in \operatorname{Pic}^{0}(X) \mid H^{i}\left(X, \omega_{X} \otimes P_{y}\right) \neq 0\right\} \\
& =\left\{y \in \operatorname{Pic}^{0}(X) \mid H^{n-i}\left(X, P_{y}^{*}\right) \neq 0\right\} .
\end{aligned}
$$

(For subsequent geometric arguments the first description is preferable, but for the present discussion the dual interpretation is easiest.) Let $y \in V_{i}(X)$ be any point, and let

$$
0 \neq v \in T_{y} \operatorname{Pic}^{0}(X)=H^{1}\left(X, \mathcal{O}_{X}\right)
$$

be a non-zero tangent vector to $\operatorname{Pic}^{0}(X)$ at $y$. One of the main themes of [GL1] is that the first order deformation theory of the groups $H^{n-i}\left(X, P_{y}^{*}\right)$ is governed by the derivative complex:

$$
H^{n-i-1}\left(X, P_{y}^{*}\right) \stackrel{\cup v}{\longrightarrow} H^{n-i}\left(X, P_{y}^{*}\right) \stackrel{\cup v}{\longrightarrow} H^{n-i+1}\left(X, P_{y}^{*}\right) .
$$

Roughly speaking, if $y \in V_{i}(X)$ is a sufficiently general point, then $v \in T_{y} \operatorname{Pic}^{0}(X)$ is tangent to $V_{i}(X)$ if and only if the two maps in (1.1) vanish, whereas if (1.1) is exact, then all the cohomology in $H^{n-i}\left(X, P_{y}^{*}\right)$ vanishes to first order in the direction of $v$. The principal result of [GL2] is that there are no higher obstructions to deforming the cohomology of topologically trivial line bundles along "straight lines" in $\mathrm{Pic}^{0}(X)$, so that a first order statement is equivalent to a global one.

More precisely, one has the following

Theorem 1.2 ([GL1], [GL2]). Fix any irreducible component

$$
S \subset V_{i}(X),
$$

and let $y \in S$ be a general point, i.e. a smooth point of $V_{i}(X)$ at which the function $h^{n-i}\left(X, P_{y}^{*}\right)$ assumes its generic value on $S$. Then:

(1.2.1) $S$ is a translate of a subtorus of $\operatorname{Pic}^{0}(X)$.

(1.2.2) $\operatorname{codim}_{P i c^{0}(X)} S \geq i-\left(\operatorname{dim} X-\operatorname{dim} \operatorname{alb}_{X}(X)\right)$.

(1.2.3) If $0 \neq v \in H^{1}\left(X, \mathcal{O}_{X}\right)$ is tangent to $S$, then the maps in (1.1) vanish, whereas if $v$ is not tangent to $S$ then (1.1) is exact.

Proof. The first assertion is Theorem 0.1 of [GL2], and the second is [GL1], Theorem 1. For (1.2.3), let $\Delta_{v}(y) \subset \operatorname{Pic}^{0}(X)$ be a neighborhood of $y$ in the "straight line" in $\operatorname{Pic}^{0}(X)$ through $y$ determined by $v$, i.e. the image of a small disk under the exponential mapping $\exp _{v}(y): \mathbf{C} \longrightarrow \operatorname{Pic}^{0}(X)$ based at $y$ in the direction $v$. Thus by (1.2.1), $\Delta_{v}(y) \subset S$ if $v$ is tangent to $S$. Corollary 3.3 of [GL2] asserts that for $t \in \Delta_{v}(y)$ in punctured neighborhood of $y$ :

$$
h^{n-i}\left(X, P_{t}^{*}\right)=\text { dimension of homology of (1.1). }
$$

Now $h^{n-i}\left(X, P_{y}^{*}\right)$ assumes its generic value at $y$, and hence if $\Delta_{v}(y) \subset S$ then

$$
h^{n-i}\left(X, P_{t}^{*}\right)=h^{n-i}\left(X, P_{y}^{*}\right)
$$

for all $t \in \Delta_{v}(y)$. It then follows from (1.2.4) that the maps in (1.1) must vanish. Similarly, if $\Delta_{v}(y) \nsubseteq S$, then the left-hand side of (1.2.4) vanishes for generic $t$ 
thanks to the fact that $S$ is an irreducible component of $V_{i}(X)$, and consequently (1.1) is exact.

Still following [GL1] and [GL2], Theorem 1.2 becomes particularly useful if it is restated via Hodge duality. After fixing a Kähler metric on $X$, Hodge theory gives conjugate linear isomorphisms:

$$
H^{n-i}\left(X, P_{y}^{*}\right) \cong H^{0}\left(X, \Omega_{X}^{n-i} \otimes P_{y}\right) .
$$

Similarly, if we represent $v \in H^{1}\left(X, \mathcal{O}_{X}\right)$ by a harmonic $(0,1)$-form, then its conjugate is a holomorphic one-form $\eta=\bar{v} \in H^{0}\left(X, \Omega_{X}^{1}\right)$, and the conjugate of (1.1) is:

$$
H^{0}\left(\Omega_{X}^{n-i-1} \otimes P_{y}\right) \stackrel{\wedge \eta}{\longrightarrow} H^{0}\left(\Omega_{X}^{n-i} \otimes P_{y}\right) \stackrel{\wedge \eta}{\longrightarrow} H^{0}\left(\Omega_{X}^{n-i+1} \otimes P_{y}\right) .
$$

Corollary 1.5. (1.5.1) Keep notation and assumptions as in Theorem 1.2, and let $\eta=\bar{v}$. Then $v$ is tangent to $S$ if and only if the maps in (1.4) vanish, and otherwise (1.4) is exact.

(1.5.2) Assume that $H^{0}\left(X, \omega_{X}\right) \neq 0$, so that that the origin $0=\left[\mathcal{O}_{X}\right] \in P i c^{0}(X)$ lies in $V_{0}(X)$. Then it is an isolated point of $V_{0}(X)$ if and only if for every non-zero $\eta \in H^{0}\left(X, \Omega_{X}^{1}\right)$, the map

$$
H^{0}\left(X, \Omega_{X}^{n-1}\right) \stackrel{\wedge \eta}{\longrightarrow} H^{0}\left(X, \Omega_{X}^{n}\right)
$$

determined by wedging with $\eta$ is surjective.

Proof. The first assertion is merely a restatement of (1.2.1), and (1.5.2) follows by taking $i=0$.

Remark 1.6. The following slight generalization will be useful. Let

$$
a: X \longrightarrow A
$$

be a holomorphic mapping from $X$ onto some complex torus $A$, and define

$$
V_{i}(X)_{A}=\left\{y \in \operatorname{Pic}^{0}(A) \mid H^{n-i}\left(X, a^{*} P_{y}\right) \neq 0\right\} .
$$

Then the evident analogues of (1.2) and (1.5) hold for these loci, where one works in $\mathrm{Pic}^{0}(A)$ instead of $\mathrm{Pic}^{0}(X)$ (so that the right-hand side of (1.2.2) involves dim $a(X)$ rather than $\left.\operatorname{dim} \operatorname{alb}_{X}(X)\right)$ and where the holomorphic one-forms that occur in (1.4) and (1.5) are the pull-backs of the flat one-forms on $A$. This is not stated explicitly in [GL1] and [GL2], but it is the natural context in which the arguments there work.

Holomorphic Euler characteristics. We assume for the remainder of this section that $X$ is a compact Kähler manifold of dimension $n$ whose Albanese mapping

$$
a=\operatorname{alb}_{X}: X \longrightarrow \operatorname{Alb}(X)=A
$$

is generically finite onto its image. It follows from [GL1, Theorem 1], or (1.2.2) above, that then

$$
h^{0}\left(X, \omega_{X} \otimes P_{y}^{*}\right)=\chi\left(X, \omega_{X}\right)
$$

for general $y \in \operatorname{Pic}^{0}(X)$. Our goal is to show that if $\chi\left(X, \omega_{X}\right)=0$, then the Albanese image of $X$ is ruled by tori. 
We start with the following useful remark due to M. Green:

Lemma 1.8. One has inclusions:

$$
\operatorname{Pic}^{0}(X) \supseteq V_{0}(X) \supseteq V_{1}(X) \supseteq \cdots \supseteq V_{n}(X)=\left\{\mathcal{O}_{X}\right\} .
$$

Proof. Suppose that $y \in V_{i}(X)(i>0)$, so that $H^{n-i}\left(X, P_{y}^{*}\right) \neq 0$. In view of (1.3), there exists a non-zero form $0 \neq \alpha \in H^{0}\left(X, \Omega_{X}^{n-i} \otimes P_{y}\right)$. It is enough to show that $\eta \wedge \alpha \neq 0 \in H^{0}\left(X, \Omega_{X}^{n-i+1} \otimes P_{y}\right)$ for general $\eta \in H^{0}\left(X, \Omega_{X}^{1}\right)$. But this follows as in [GL1, end of proof of Theorem 2.10]. In brief, fix a general point $x \in X$ at which $\alpha(x) \neq 0$. Since $X$ has maximal Albanese dimension, if we have chosen $x$ sufficiently generally, we can find holomorphic one-forms $\eta_{1}, \ldots, \eta_{n} \in H^{0}\left(X, \Omega_{X}^{1}\right)$ such that the $\eta_{i}(x)$ form a basis of the holomorphic cotangent space $T_{x}^{*} X$. But then it is immediate that $\alpha(x) \wedge \eta_{j}(x) \neq 0$ for some $j \in[1, n]$.

We now turn to the demonstration of Theorem 3. As noted in the Introduction, the argument that follows builds substantially on discussions with M. Green some years ago.

Proof of Theorem 3. Assume that $\chi\left(X, \omega_{X}\right)=0$. Then by $(1.7), V_{0}(X)$ is a proper subvariety of $\operatorname{Pic}^{0}(X)$. Fix an irreducible component $S \subset V_{0}(X)$ and a general point $y \in S$, and put

$$
k=\operatorname{codim}_{\mathrm{Pic}^{0}(X)} S .
$$

Note that it follows from (1.2.2) that $S$ cannot be contained in $V_{j}(X)$ for $j>$ $k$. By contrast, if $S \subseteq V_{j}(X)$ for some $j \leq k$, then in fact $S$ is an irreducible component of $V_{j}(X)$ thanks to the previous lemma. Therefore, by taking $y \in S$ sufficiently generally, we may suppose that $H^{n-j}\left(X, P_{y}^{*}\right)=0$ for $j>k$, and that if $H^{n-j}\left(X, P_{y}^{*}\right) \neq 0$ for $j \leq k$, then the conclusions of Theorem 1.2 hold with $i=j$ at $y$. In particular, it follows from (1.2.3) that if $0 \neq v \in H^{1}\left(X, \mathcal{O}_{X}\right)=T_{y} \operatorname{Pic}^{0}(X)$ is not tangent to $S$ at $y$, then the derivative complex

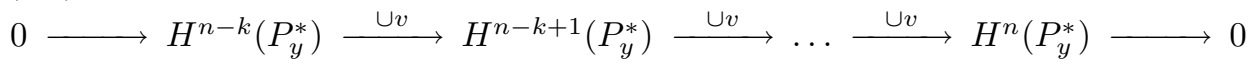

is (everywhere) exact.

We claim that

$$
H^{n-k}\left(X, P_{y}^{*}\right) \neq 0,
$$

i.e. that $S$ is actually a component of $V_{k}(X)$ (and hence also that $k \leq n$ ). In fact, let

$$
V \subset H^{1}\left(X, \mathcal{O}_{X}\right)=T_{y} \operatorname{Pic}^{0}(X)
$$

be a $k$-dimensional subspace complementary to $T_{y} S \subset T_{y} \mathrm{Pic}^{0}(X)$ (so that $V$ represents the normal space to $S$ at $y$.) Thus (1.9) is exact for each $0 \neq v \in V$. Set $\mathbf{P}=\mathbf{P}\left(V^{*}\right)$, so that $\mathbf{P}$ is a projective space of dimension $k-1$. Then we may assemble the derivative complexes (1.9) determined by $0 \neq v \in V$ into a complex $K$ of vector bundles on $\mathbf{P}$ :

$$
\begin{aligned}
0 \rightarrow H^{n-k}\left(P_{y}^{*}\right) \otimes \mathcal{O}_{\mathbf{P}}(-k) & \rightarrow H^{n-k+1}\left(P_{y}^{*}\right) \otimes \mathcal{O}_{\mathbf{P}}(-k+1) \\
& \rightarrow \cdots \rightarrow H^{n}\left(P_{y}^{*}\right) \otimes \mathcal{O}_{\mathbf{P}} \rightarrow 0 .
\end{aligned}
$$


The fact that each of the point-wise complexes (1.9) is exact implies that $K$ is exact as a complex of sheaves on $\mathbf{P}$. It then follows by chasing through $K$ and taking cohomology on $\mathbf{P}$ that

$$
0 \neq H^{n}\left(X, P_{y}^{*}\right) \cong H^{n-k}\left(X, P_{y}^{*}\right),
$$

and (1.10) is established. [Compare [Mori], Proof of (3.3.2).]

We now argue as in [GL2], $\S 4$. Recalling that $S$ is (a translate of) a subtorus of $\operatorname{Pic}^{0}(X)$, let $C=S^{*}$ be the dual torus. Since $A=\operatorname{Alb}(X)$ is the dual of $\operatorname{Pic}^{0}(X)$, the inclusion $S \hookrightarrow \operatorname{Pic}^{0}(X)$ determines a quotient map

$$
\pi: A=\operatorname{Alb}(X) \longrightarrow C
$$

whose fibres are translates of the $k$-dimensional connected subtorus

$$
B=_{\text {def }} \operatorname{ker}(\pi) .
$$

Let $Y=\operatorname{alb}_{X}(X) \subset A$ be the Albanese image of $X$, and let

$$
\begin{gathered}
g: X \stackrel{\operatorname{alb}_{X}}{\longrightarrow} Y \subset A \stackrel{\pi}{\longrightarrow} C \\
h: Y \subset A \stackrel{\pi}{\longrightarrow} C
\end{gathered}
$$

denote the indicated compositions. We claim that

$$
\operatorname{dim} g(X) \leq n-k .
$$

Grant this for a moment. Since $a: X \longrightarrow Y$ is generically finite and surjective, it then follows that all the fibres of $h: Y \longrightarrow h(Y) \subset C$ have dimension $\geq k$. But these fibres are contained in translates of the $k$-dimensional torus $B$. In other words, the fibres of $Y \longrightarrow h(Y)$ fill up the fibres of $A \longrightarrow C$ over $h(Y)$. Therefore $Y$ is ruled by tori, as was to be shown.

It remains only to prove (1.12). But this is in fact established in [GL2, p. 92]. We summarize the argument for the convenience of the reader. Let

$$
v_{1}, \ldots, v_{q-k} \in H^{1}\left(X, \mathcal{O}_{X}\right)=T_{y} S
$$

be a basis for the tangent space to $S$ at $y$, where $q=\operatorname{dim} \operatorname{Pic}^{0}(X)$. As in the previous subsection, let $\eta_{i}=\bar{v}_{i} \in H^{0}\left(X, \Omega_{X}^{1}\right)$ be conjugate holomorphic one-forms. The map $g: X \longrightarrow C$ arises by integrating the $\eta_{i}$, and consequently for a general point $x \in X$ :

$$
\operatorname{dim} g(X)=\operatorname{dim} \operatorname{span}\left\{\eta_{1}(x), \ldots, \eta_{q-k}(x)\right\} \subset T_{x}^{*} X .
$$

Since $\eta_{i}$ is the conjugate of a tangent vector to $S$, it follows from (1.5.1) that each of the maps

$$
H^{0}\left(X, \Omega_{X}^{n-k} \otimes P_{y}\right) \stackrel{\wedge \eta_{i}}{\longrightarrow} H^{0}\left(X, \Omega_{X}^{n-k+1} \otimes P_{y}\right)
$$

vanishes. But the group on the left in $\left(^{*}\right)$ is non-zero thanks to (1.10). An elementary pointwise calculation (cf. [GL2], Lemma 4.1) shows that the space of one-forms that annihilates a non-zero $n-k$ form has dimension $\leq n-k$, and hence (1.12) follows.

We conclude this section by giving an example to show that Kollár's conjecture [Kol3, (17.9)] fails in general. 
Example 1.13. We exhibit a smooth variety $X$ of general type having maximal Albanese dimension such that $\chi\left(X, \omega_{X}\right)=0$. Let $E$ be an elliptic curve, and $p: C \longrightarrow E$ a double covering of $E$ by a curve $C$ of genus $\geq 2$. Denote by $\iota: C \longrightarrow C$ the corresponding involution of $C$. Let $A=E \times E \times E, V=C \times C \times C$, and consider the involution $\tau=\iota \times \iota \times \iota$ of $V$. Set $Y=V /\{1, \tau\}$. The spaces in question fit into a tower of Galois covers:

$$
V \stackrel{f}{\longrightarrow} Y \stackrel{g}{\longrightarrow} A
$$

of degrees 2 and 4 respectively. Observe that $Y$ is smooth except at finitely many points at which it is locally analytically isomorphic to a Veronese cone, i.e. the quotient of $\mathbf{C}^{3}$ by multiplication by -1 . In particular $Y$ has only terminal and hence rational singularities. The map $f: V \longrightarrow Y$ being étale off the finitely many singular points of $Y$, we see that $Y$ is of general type, and in fact minimal of global index two. Let

$$
h: X \longrightarrow Y
$$

be a resolution of singularities. Clearly $X$ has maximal Albanese dimension, and we claim that $\chi\left(X, \omega_{X}\right)=0$. In order to verify this, it suffices in view of (1.7) to show that $H^{3}\left(X, h^{*} g^{*} P\right)=0$ for a general topologically trivial line bundle $P \in \operatorname{Pic}^{0}(A)$. To this end, start with non-trivial line bundles $P_{1}, P_{2}, P_{3} \in \operatorname{Pic}^{0}(E)$, and take $P$ to be their exterior product. Recalling that $Y$ has only rational singularities, one finds:

$$
H^{3}\left(X, h^{*} g^{*} P\right)=H^{3}\left(Y, g^{*} P\right)=H^{3}\left(V, f^{*} g^{*} P\right)^{\{1, \tau\}} .
$$

By Künneth:

$$
H^{3}\left(V, f^{*} g^{*} P\right)=H^{1}\left(C, p^{*} P_{1}\right) \otimes H^{1}\left(C, p^{*} P_{2}\right) \otimes H^{1}\left(C, p^{*} P_{3}\right),
$$

and since each $P_{i}$ is non-trivial, $\iota$ acts by -1 on each factor. Hence

$$
H^{3}\left(V, f^{*} g^{*} P\right)^{\{1, \tau\}}=0,
$$

as required. [Alternatively, as Kollár notes, one can compute $(g \circ h)_{*}\left(\omega_{X}\right)$.]

\section{VARIETIES OF Kodaira Dimension ZeRo}

We give in this section the applications to varieties of Kodaira dimension zero. Denote by $X$ a smooth projective variety of dimension $n$, and write $P_{m}(X)$ for the $m^{\text {th }}$ plurigenus of $X$, i.e. $P_{m}(X)=h^{0}\left(X, \omega_{X}^{\otimes m}\right)$. As in $\S 1$ we consider the loci:

$$
V_{i}(X)=\left\{y \in \operatorname{Pic}^{0}(X) \mid H^{i}\left(X, \omega_{X} \otimes P_{y}\right) \neq 0\right\} .
$$

We emphasize that we do not assume here that the Albanese mapping of $X$ is generically finite.

Theorem 4 from the Introduction is an immediate consequence of two elementary general propositions:

Proposition 2.1. If $P_{1}(X)=P_{2}(X)=1$, then the origin is an isolated point of $V_{0}(X)$.

Proposition 2.2. If the origin is an isolated point of $V_{0}(X)$, then the Albanese mapping $a b_{X}: X \longrightarrow A l b(X)$ is surjective. 
Proof of Proposition 2.1. Since $P_{1}(X) \neq 0$, the origin $\mathcal{O}_{X}$ lies in $V_{0}(X)$. Suppose that it is not an isolated point. Then by (1.2.1), $V_{0}(X)$ contains a subgroup $S \subset$ $V_{0}(X)$ of positive dimension. In particular, if $y \in S$ then also $-y \in S$, and therefore for each $y \in S$ the image of

$$
H^{0}\left(X, \omega_{X} \otimes P_{y}\right) \otimes H^{0}\left(X, \omega_{X} \otimes P_{y}^{*}\right) \longrightarrow H^{0}\left(X, \omega_{X}^{\otimes 2}\right)
$$

is non-zero. Since a given divisor in the linear series $\left|\omega_{X}^{\otimes 2}\right|$ has only finitely many irreducible components, it follows that as $y$ varies over the positive dimensional torus $S$, the image of $(*)$ must vary as well. Therefore $P_{2}(X)>1$, a contradiction. [Compare the proof of [Kol3], Theorem 17.10.]

Proof of Proposition 2.2. Assume that the origin is an isolated point of $V_{0}(X)$, but that $\operatorname{alb}_{X}$ is not surjective. Fix an arbitrary point $x \in X$. The non-surjectivity of $\mathrm{alb}_{X}$ implies that there exists a non-zero holomorphic one-form

$$
0 \neq \eta=\eta_{x} \in H^{0}\left(X, \Omega_{X}^{1}\right) \text { such that } \eta(x)=0 .
$$

[Take $\eta$ to be the pull-back of a flat one-form on $\operatorname{Alb}(X)$ lying in the kernel of the coderivative $T_{\operatorname{alb}(x)}^{*} \operatorname{Alb}(X) \longrightarrow T_{x}^{*} X$ of $\operatorname{alb}_{X}$ at $x$.] On the other hand, since the origin is an isolated point of $V_{0}(X)$, it follows from (1.5.2) that wedging with $\eta_{x}$ gives a surjective map

$$
H^{0}\left(X, \Omega_{X}^{n-1}\right) \stackrel{\wedge \eta_{x}}{\longrightarrow} H^{0}\left(X, \Omega_{X}^{n}\right) .
$$

Therefore every section of $\omega_{X}=\Omega_{X}^{n}$ vanishes at (the arbitrary point) $x$, and hence $H^{0}\left(X, \omega_{X}\right)=0$, a contradiction.

Remark 2.3. For the convenience of the reader, we recall the standard argument showing that Theorem 4 implies Kawamata's result that if $Y$ is a smooth projective variety with $\kappa(Y)=0$, then alb $_{Y}$ is surjective. In fact, by a lemma of Fujita (cf. [Mori], (4.1)), there exists a smooth projective variety $X$ of Kodaira dimension zero, admitting a generically finite surjective map $f: X \longrightarrow Y$, such that $P_{1}(X) \neq 0$. Since $\kappa(X)=0$, it follows that $P_{1}(X)=P_{2}(X)=1$. Therefore alb ${ }_{X}$ is surjective thanks to Theorem 4 , and this easily implies that alb $b_{Y}$ is surjective.

In the remainder of this section, we show how similar ideas lead to new proofs of some other results concerning varieties of Kodaira dimension zero. We start with a theorem of Kollár [Kol1] giving a birational characterization of abelian varieties. Kollár's statement was in turn an effective version of a theorem of KawamataViehweg $[\mathrm{KV}]$ characterizing abelian varieties birationally as projective manifolds with $\kappa=0$ and $q=n$. Some stronger results appear in [Kol2] and [Kol3], but it is not clear whether one could recover them by these techniques.

Proposition 2.4 (Kollár, [Kol1]). Let $X$ be a smooth projective variety of dimension $n$, and assume that

$$
P_{1}(X)=P_{4}(X)=1 \quad \text { and } \quad q(X)=\operatorname{def} h^{1}\left(X, \mathcal{O}_{X}\right)=n .
$$

Then $X$ is birational to an abelian variety.

Proof. The beginning of the argument follows Kollár's proof. The Albanese mapping

$$
a=\operatorname{alb}_{X}: X \longrightarrow \operatorname{Alb}(X)=A
$$


is surjective by Theorem 4 , hence generically finite since $q=\operatorname{dim} A=\operatorname{dim} X=$ $n$. If $a$ is a birational isomorphism, we are done. Otherwise, since the Albanese map does not factor through any étale covers of $A$, it follows by considering the Stein factorization of $a$ that the ramification divisor of $a$ must contain at least one component $\Delta$ which maps to a divisor $D \subset A$. Replacing $X$ if necessary by a suitable blow-up, we may assume that $\Delta$ is smooth. Note that since the ramification divisor $\operatorname{Ram}(a)$ represents $\omega_{X}$, there is a natural inclusion $\omega_{X}(\Delta) \hookrightarrow \omega_{X}^{\otimes 2}$.

We now apply (1.2) and (2.2) to $\Delta$. Specifically, since $a(\Delta)=D$ is a divisor in $A$, we have $P_{1}(\Delta) \geq 1$. Suppose first that $D$ spans $A$. Then evidently alb $\Delta$ is not surjective. Proposition 2.2 implies that $\mathcal{O}_{\Delta}$ is not an isolated point of $V_{0}(\Delta)$, which therefore contains a subtorus of positive dimension. In fact, combining Remark 1.6 (applied to $\Delta$ ) with the proof of Proposition 2.2, we see that there is a positive dimensional subgroup $S_{\Delta} \subset \operatorname{Pic}^{0}(X)$ such that

$$
H^{0}\left(\Delta, \omega_{\Delta} \otimes P_{y}\right) \neq 0 \quad \forall y \in S_{\Delta} .
$$

The hypotheses on the plurigenera of $X$ force $P_{2}(X)=1$, and so Proposition 2.1 implies that $\mathcal{O}_{X}$ is an isolated point of $V_{0}(X)$. Therefore, thanks to Lemma 1.8, $\mathcal{O}_{X}$ is an isolated point of all the $V_{i}(X)$, and in particular

$$
H^{0}\left(X, \omega_{X} \otimes P_{y}\right)=H^{1}\left(X, \omega_{X} \otimes P_{y}\right)=0
$$

for $y$ in punctured neighborhood of 0 in $S_{\Delta}$. In view of (2.4.1), it follows from the exact sequence

$$
0 \longrightarrow \omega_{X} \otimes P_{y} \longrightarrow \omega_{X}(\Delta) \otimes P_{y} \longrightarrow \omega_{\Delta} \otimes P_{y} \longrightarrow 0
$$

that $H^{0}\left(X, \omega_{X}(\Delta) \otimes P_{y}\right) \neq 0$ for every $y \in S_{\Delta}$. Then just as in the proof of Proposition 2.1, this implies that

$$
h^{0}\left(X, \omega_{X}^{\otimes 2}(2 \Delta)\right) \geq 2 .
$$

But $H^{0}\left(X, \omega_{X}^{\otimes 2}(2 \Delta)\right) \subset H^{0}\left(X, \omega_{X}^{\otimes 4}\right)$, and hence $P_{4}(X)>1$, a contradiction. It remains to treat the possibility that $\Delta$ maps to a codimension one subtorus $D \subset$ $A$, but in this case it is enough to take $S_{\Delta}$ to be the kernel of the natural map $\operatorname{Pic}^{0}(X)=\operatorname{Pic}^{0}(A) \longrightarrow \operatorname{Pic}^{0}(\Delta)$.

We conclude this section by showing how similar ideas lead to the more precise result from $[\mathrm{Ka}]$ that the Albanese mapping of a variety of Kodaira dimension zero is a fibre space, i.e. has connected fibres:

Proposition 2.5 (Kawamata $[\mathrm{Ka}]$ ). Let $X$ be a smooth projective variety of Kodaira dimension zero. Then the fibres of the Albanese mapping

$$
a=a l b_{X}: X \longrightarrow A l b(X)=A
$$

are connected.

Proof. There is no loss in generality in replacing $X$ with a birationally equivalent model. So by starting with the Stein factorization of $a$ and resolving singularities and indeterminacies, we can assume that $a=\operatorname{alb}_{X}$ admits a factorization:

$$
X \stackrel{g}{\longrightarrow} V \stackrel{b}{\longrightarrow} A,
$$

where $g$ is surjective with connected fibres, $V$ is smooth and projective, and $b$ is generically finite. We know already (e.g. by Theorem 4 and Remark 2.3) that $a$ is surjective, and hence $b$ is a generically finite covering. We assume by way of contradiction that $b$ has degree $>1$. Since the Albanese mapping does not factor 
through any étale coverings of $A, b$ cannot be birationally étale, and $V$ cannot be birational to an abelian variety. Therefore $b$ has a non-trivial ramification divisor $R$, and by Proposition 2.4, $P_{4}(V)>1$.

We next reduce in effect to the situation $P_{1} \neq 0$. In fact, by Fujita's lemma [Mori, (4.1)] there are a smooth projective variety $Y$, and a generically finite surjective covering $\nu: Y \longrightarrow X$ such that $\kappa(Y)=\kappa(X)=0$ and $P_{1}(Y) \neq 0$. Therefore $P_{1}(Y)=1$, and we denote by $K_{Y}$ the unique effective canonical divisor on $Y$. We put $f=g \circ \nu$, and consider the maps:

$$
Y \stackrel{f}{\longrightarrow} V \stackrel{b}{\longrightarrow} A .
$$

Let $\Delta \subset V$ be any irreducible component of the ramification divisor $R=\operatorname{Ram}(b)$. We claim:

$$
\text { Any irreducible component } D \text { of } f^{*}(\Delta) \text { appears in } K_{Y} \text {. }
$$

Grant this for the time being. We can write

$$
f^{*}(R)=\sum a_{i} D_{i} \quad\left(a_{i}>0\right),
$$

where the $D_{i}$ are distinct irreducible divisors, and $\sum D_{i} \prec K_{Y}$ by (2.5.1). Let $a=\max \left\{a_{i}\right\}$. Then for any $m>0$ :

$$
\begin{aligned}
f^{*}(m R)=\sum m a_{i} D_{i} & \prec \sum m a D_{i} \\
& \prec m a K_{Y} .
\end{aligned}
$$

On the other hand, since $b: V \longrightarrow A$ is a generically finite covering of an abelian variety, one has $R \equiv K_{V}$. Therefore

$$
P_{4}(V)=h^{0}\left(V, \mathcal{O}_{V}(4 R)\right) \geq 2,
$$

and hence $h^{0}\left(Y, \mathcal{O}_{Y}\left(4 a K_{Y}\right)\right) \geq 2$ thanks to $\left(^{*}\right)$. But $\kappa(Y)=0$, so this is a contradiction.

It remains to prove (2.5.1). Let $v \in \Delta$ be any point, and $y \in f^{-1}(v)$. Since $b$ ramifies at $v$, as in the proof of Proposition 2.2 there is a homolorphic one-form

$$
0 \neq \eta_{v} \in H^{0}\left(V, \Omega_{V}^{1}\right) \text { such that } \eta_{v}(v)=0 .
$$

On the other hand, Proposition 2.1 and the fact that $\kappa(Y)=0$ imply that $\mathcal{O}_{Y}$ is an isolated point of $V_{0}(Y)$. It follows from (1.5.2) that the map

$$
H^{0}\left(Y, \Omega_{Y}^{n-1}\right) \stackrel{\wedge\left(f^{*} \eta_{v}\right)}{\longrightarrow} H^{0}\left(Y, \Omega_{Y}^{n}\right)
$$

is surjective. But $f^{*} \eta_{v}(y)=0$, and hence $K_{Y}$ vanishes at $y$.

Remark 2.6. Propositions 2.1, 2.2 and 2.4 extend with no difficulties to the setting of compact Kähler manifolds. In the proof of (2.5) one has to take a little care that one can arrange for $V$ to be Kähler, and as the referee points out for this one can apply [Var], Theorem 3 .

\section{Singularities of theta Divisors}

We start with some preliminaries on adjoint ideals, and then give the applications to theta divisors. The reader who wishes to read this section independently of the rest of the paper is referred to Remark 3.4. 
Adjoint ideals. We wish to understand how the adjunction formula works for a possibly singular divisor in a smooth variety. The following Proposition generalizes various classical constructions involving conductor ideals. The result is certainly at least implicitly known to the experts, but we include a proof for the benefit of the reader.

Proposition 3.1. Let $M$ be a smooth variety, let $D \subset M$ be a reduced effective divisor, and let $f: X \longrightarrow D$ be any resolution of singularities. Then there is an adjoint ideal

$$
\mathcal{J}=\mathcal{J}_{D} \subset \mathcal{O}_{M}
$$

cosupported in the singular locus of $D$, which sits in an exact sequence:

$$
0 \longrightarrow \mathcal{O}_{M}\left(K_{M}\right) \stackrel{\cdot D}{\longrightarrow} \mathcal{O}_{M}\left(K_{M}+D\right) \otimes \mathcal{J} \longrightarrow f_{*} \mathcal{O}_{X}\left(K_{X}\right) \longrightarrow 0 .
$$

Moreover, $\mathcal{J}=\mathcal{O}_{M}$ if and only if $D$ is normal and has only rational singularities.

Proof. Note to begin with that the sheaf $f_{*} \omega_{X}$ is independent of the choice of resolution, so we are free to work with any convenient one. Let $g: Y \longrightarrow M$ be an embedded resolution of $D$, and let $X \subset Y$ be the proper transform of $D$ (so $X$ is a disjoint union of smooth divisors). Then we can write

$$
K_{Y}+X=g^{*}\left(K_{M}+D\right)+P-N,
$$

where $P, N$ and $X$ are effective divisors on $Y$, with no common components, and every component of $P$ is $g$-exceptional. The adjoint ideal $\mathcal{J}=\mathcal{J}_{D}$ is defined to be

$$
\mathcal{J}=g_{*} \mathcal{O}_{Y}(-N) \subseteq g_{*} \mathcal{O}_{Y}=\mathcal{O}_{M} .
$$

Since $P$ is $g$-exceptional, one sees that $g_{*} \mathcal{O}_{Y}(P-N)=g_{*} \mathcal{O}_{Y}(-N)$ (or cf. [KMM, 1.3.2]). We then have:

$$
\begin{aligned}
g_{*} \mathcal{O}_{Y}\left(K_{Y}+X\right) & =\mathcal{O}_{X}\left(K_{M}+D\right) \otimes g_{*} \mathcal{O}_{Y}(P-N) \\
& =\mathcal{O}_{X}\left(K_{M}+D\right) \otimes g_{*} \mathcal{O}_{Y}(-N) \\
& =\mathcal{O}_{X}\left(K_{M}+D\right) \otimes \mathcal{J} .
\end{aligned}
$$

Recalling that $g_{*} \mathcal{O}_{Y}\left(K_{Y}\right)=\mathcal{O}_{M}\left(K_{M}\right)$ and $R^{1} g_{*} \mathcal{O}_{Y}\left(K_{Y}\right)=0,(3.1 .1)$ arises as the pushforward under $g$ of the exact sequence

$$
0 \longrightarrow \mathcal{O}_{Y}\left(K_{Y}\right) \longrightarrow \mathcal{O}_{Y}\left(K_{Y}+X\right) \longrightarrow \mathcal{O}_{X}\left(K_{X}\right) \longrightarrow 0 .
$$

It follows from (3.1.1) that $\mathcal{J}_{D}=\mathcal{O}_{M}$ iff $f_{*} \omega_{X}=\omega_{D}$. Since $f$ factors through the normalization of $D$, and since $D$ is non-normal iff it is singular in codimension one, this can evidently hold only if $D$ is normal. And as $D$ is in any event CohenMacaulay, the equality in question is then equivalent to the condition that $D$ has at worst rational singularities (cf. [Kol4, (11.10)]).

Remark 3.2. As in the Introduction, consider the multiplicity loci

$$
\Sigma_{k}(D)=\left\{x \in M \mid \operatorname{mult}_{x}(D) \geq k\right\} .
$$

We observe that if $\Sigma_{k}(D)$ has any components of codimension $\leq k$ in $M$, for some $k \geq 2$, then the corresponding adjoint ideal $\mathcal{J}=\mathcal{J}_{D}$ is non-trivial. In fact, construct the embedded resolution $Y$ by first blowing up such a component. Then one sees that the divisor $N$ appearing in the proof of (3.1) is non-zero, and the assertion follows. (Compare [Kol3], proof of Theorem 17.13.) 
Theta divisors. Let $(A, \Theta)$ be a principally polarized abelian variety. We start with the following statement, which in view of (3.1) is equivalent to Theorem 1 from the Introduction.

Theorem 3.3. Assume that $\Theta$ is irreducible. Then the corresponding adjoint ideal is trivial, i.e. $\mathcal{J}=\mathcal{J}_{\Theta}=\mathcal{O}_{A}$.

Proof. We may assume that $g=\operatorname{dim} A \geq 2$, for otherwise the statement is trivial. Let $f: X \longrightarrow \Theta$ be a resolution. Note first that $X$ (i.e. $\Theta$ ) is of general type. Otherwise, by a theorem of Ueno (cf. [Mori], (3.7)) there would exist a non-trivial quotient $\pi: A \longrightarrow B$ of $A$, plus an effective divisor $E \subset B$ such that $\Theta \subset \pi^{*}(E)$. But this is impossible since $\Theta$ is ample. [We remark that Ueno's theorem is the essential point where irreducibility is used: for $\Theta$ reducible, the individual components won't be of general type.]

The adjoint exact sequence (3.1.1) takes the form:

$$
0 \longrightarrow \mathcal{O}_{A} \longrightarrow \mathcal{O}_{A}(\Theta) \otimes \mathcal{J} \longrightarrow f_{*} \omega_{X} \longrightarrow 0 .
$$

Now let $P \in \operatorname{Pic}^{0}(A)$ be a topologically trivial line bundle on $A$, and twist (3.3.1) by $P$ :

$$
0 \longrightarrow P \longrightarrow \mathcal{O}_{A}(\Theta) \otimes P \otimes \mathcal{J} \longrightarrow f_{*} \omega_{X} \otimes P \longrightarrow 0 .
$$

Evidently $X$ has maximal Albanese dimension. Bearing in mind Remark 1.6, it follows from [GL1, Theorem 1] (or (1.2.2) above) that $H^{i}\left(X, \omega_{X} \otimes f^{*} P\right)=0$ for $i>0$ and $P$ general. Therefore

$$
\begin{aligned}
H^{0}\left(A, f_{*} \omega_{X} \otimes P\right) & =H^{0}\left(X, \omega_{X} \otimes f^{*} P\right) \\
& =\chi\left(X, \omega_{X} \otimes f^{*} P\right) \\
& =\chi\left(X, \omega_{X}\right)
\end{aligned}
$$

for generic $P$. But by construction $X$ is birational to its Albanese image, and hence $\chi\left(X, \omega_{X}\right) \neq 0$ thanks to Theorem 3. Thus $H^{0}\left(A, f_{*} \omega_{X} \otimes P\right) \neq 0$ for general $P$. Let $\alpha_{P} \in A$ be the point corresponding to $P \in \operatorname{Pic}^{0}(A)$ under the isomorphism $A \cong \operatorname{Pic}^{0}(A)$ determined by the principal polarization $\mathcal{O}_{A}(\Theta)$. Then (3.3.2) implies that

$$
H^{0}\left(A, \mathcal{O}_{A}\left(\Theta+\alpha_{P}\right) \otimes \mathcal{J}\right)=H^{0}\left(A, \mathcal{O}_{A}(\Theta) \otimes P \otimes \mathcal{J}\right) \neq 0
$$

for general $P \in \operatorname{Pic}^{0}(A)$ (and hence general $\alpha_{P} \in A$ ).

Suppose now that the theorem is false, so that $\mathcal{J} \neq \mathcal{O}_{A}$. Then the corresponding zero-locus

$$
Z=\operatorname{Zeroes}(\mathcal{J}) \subset A
$$

is non-empty. Since $h^{0}\left(A, \mathcal{O}_{A}\left(\Theta+\alpha_{P}\right)\right)=1$, it follows from (3.3.3) that $Z \subseteq(\Theta+\alpha)$ for a general point $\alpha \in A$. But the translates of $\Theta$ don't have any points in common, so this is impossible.

Remark 3.4. We indicate how to avoid the appeal to Theorem 3 (and Remark 1.6) in the argument just completed. Keeping notation as above, Theorem 3 was invoked only in order to show that $\chi\left(X, \omega_{X}\right) \neq 0$. This can be circumvented by noting from (3.3.1) that $H^{0}\left(X, f_{*} \omega_{X}\right) \subseteq H^{1}\left(A, \mathcal{O}_{A}\right)$, and hence $p_{g}(X) \leq g=$ $\operatorname{dim} A$. But then a theorem of Kawamata and Viehweg [KV] (cf. [Mori, (3.11)]) implies that $\chi\left(X, \omega_{X}\right)=1$. In fact, Kawamata and Viehweg prove that the maps $H^{0}\left(A, \Omega_{A}^{i}\right) \longrightarrow H^{0}\left(X, \Omega_{X}^{i}\right)$ are isomorphisms for $i \leq g-1$, and taking $i=1$ it 
follows that $\operatorname{Pic}^{0}(A) \longrightarrow \mathrm{Pic}^{0}(X)$ is an isogeny. Hence one can also bypass Remark 1.6: it is essentially the same to work with general $P \in \operatorname{Pic}^{0}(X)$ (as in [GL1]) or with general $P \in \operatorname{Pic}^{0}(A)$ (as in the argument above).

Next we give the

Proof of Corollary 2. Assume that there exists an integer $k \geq 2$ such that $\Sigma_{k}(\Theta)$ has an irreducible component of codimension $k$ in $A$. We wish to show that then $(A, \Theta)$ is a non-trivial $k$-fold product of principally polarized abelian varieties. It follows in the first place from Remark 3.2 that $\mathcal{J}_{\Theta} \neq \mathcal{O}_{A}$. Therefore $\Theta$ is reducible by Theorem 3.3. The Decomposition Theorem (cf. [LB, (4.3.1)]) then implies that the p.p.a.v. $(A, \Theta)$ splits as a non-trivial product. Let

$$
(A, \Theta)=\left(A_{1}, \Theta_{1}\right) \times \cdots \times\left(A_{r}, \Theta_{r}\right)
$$

be the decomposition of $(A, \Theta)$ as a product of irreducible p.p.a.v.'s. Given any irreducible subset $S \subset \Sigma_{k}(\Theta)$, there exist integers $k_{1}, \ldots, k_{r} \geq 0$, with $\sum k_{i} \geq k$, such that

$$
S \subseteq \Sigma_{k_{1}}\left(\Theta_{1}\right) \times \cdots \times \Sigma_{k_{r}}\left(\Theta_{r}\right) .
$$

Suppose that $\operatorname{codim}_{A} S=k$. Since in any event $\operatorname{codim}_{A_{i}} \Sigma_{k_{i}}\left(\Theta_{i}\right) \geq k_{i}$ by Kollár's theorem, it follows that

$$
\begin{aligned}
k=\operatorname{codim}_{A} S & \geq \operatorname{codim}_{A_{1}} \Sigma_{k_{1}}\left(\Theta_{1}\right)+\cdots+\operatorname{codim}_{A_{r}} \Sigma_{k_{r}}\left(\Theta_{r}\right) \\
& \geq k_{1}+\cdots+k_{r} \\
& \geq k
\end{aligned}
$$

Therefore

$$
\operatorname{codim}_{A_{i}} \Sigma_{k_{i}}\left(\Theta_{i}\right)=k_{i} \text { for all } i .
$$

By induction, this implies that $k_{i}=0$ or 1 for all $i$. But then it follows from $\left(^{*}\right)$ that $r \geq k$.

Finally, we present an extension of Kollár's theorem to the singularities of pluritheta divisors, as proposed in [Kol3, Problem 17.15].

Proposition 3.5. Let $(A, \Theta)$ be a p.p.a.v., and for $m \geq 1$ fix any divisor $D \in$ $|m \Theta|$. Then the pair $\left(A, \frac{1}{m} D\right)$ is log-canonical. In particular, every component of $\Sigma_{m k}(D)$ has codimension $\geq k$ in $A$.

Proof. The point of the argument is to use systematically all the available vanishings. Specifically, for $0<\epsilon \ll 1$ consider the divisor

$$
E=E_{\epsilon}=\frac{1-m \epsilon}{m} D \equiv(1-\epsilon) \Theta .
$$

Thus $\Theta-E$ is an ample Q-divisor, and hence so is $P(\Theta-E)$ for any $P \in \operatorname{Pic}^{0}(A)$. Denote by $\mathcal{J}=\mathcal{J}_{E} \subset \mathcal{O}_{A}$ the multiplier ideal determined by $E$ (cf. [De, (6.12)] or $[$ Kol4, (2.16)]). Then Kawamata-Viehweg-Nadel vanishing implies that

$$
H^{i}\left(A, \mathcal{O}_{A}(\Theta) \otimes P \otimes \mathcal{J}\right)=0 \text { for all } i>0 \text { and } P \in \operatorname{Pic}^{0}(A) .
$$

Assuming the assertion of the Proposition false, we can choose $\epsilon \ll 1$ such that $\mathcal{J} \neq \mathcal{O}_{A}$. Then $Z=\operatorname{Zeroes}(\mathcal{J}) \neq \emptyset$, and as in the proof of Theorem 3.3 it follows 
that

$$
H^{0}\left(A, \mathcal{O}_{A}(\Theta) \otimes P \otimes \mathcal{J}\right)=0
$$

for general $P \in \operatorname{Pic}^{0}(A)$. Combining (3.5.1) and (3.5.2), we find that

$$
\chi\left(A, \mathcal{O}_{A}(\Theta) \otimes P \otimes \mathcal{J}\right)=0
$$

for general $P$. As Euler characteristics are deformation invariants, this then holds for arbitrary $P \in \operatorname{Pic}^{0}(A)$. Thanks to (3.5.1), the equality (3.5.2) must likewise hold for all $P$. In other words:

$$
H^{i}\left(A, \mathcal{O}_{A}(\Theta) \otimes P \otimes \mathcal{J}\right)=0 \text { for all } i \geq 0 \text { and all } P \in \operatorname{Pic}^{0}(A) .
$$

But it follows from Mukai's theory [Muk] of the Fourier functor on an abelian variety that if $F$ is a coherent sheaf on $A$ such that $H^{i}(A, F \otimes P)=0$ for all $i \geq 0$ and all $P \in \operatorname{Pic}^{0}(A)$, then $F=0$. Thus we have a contradiction.

\section{REFERENCES}

[AM] A. Andreotti and A. Mayer, On period relations for abelian integrals on algebraic curves, Ann. Scuola Norm. Sup. Pisa 21 (1967), 189-238. MR 36:3792

[AD] E. Arbarello and C. DeConcini, Another proof of a conjecture of S. P. Novikov on periods of abelian integrals on Riemann surfaces, Duke Math. J. 54 (1987), 163-178. MR 88i: 14025

[De] J.-P. Demailly, $L^{2}$ vanishing theorems for positive line bundles and adjunction theory (to appear).

[GL1] M. Green and R. Lazarsfeld, Deformation theory, generic vanishing theorems, and some conjectures of Enriques, Catanese and Beauville, Invent. Math. 90 (1987), 389-407. MR 89b:32025

[GL2] Higher obstructions to deforming cohomology groups of line bundles, Jour. of A.M.S. 4 (1991), 87-103. MR 92i:32021

[Ka] Y. Kawamata, Characterization of abelian varieties, Comp. Math. 43 (1981), 253-276. MR 83j: 14029

[KMM] Y. Kawamata, K. Matsuda and K. Matsuki, Introduction to the minimal model program, Adv. Study in Pure Math. 10 (1987), 283-360. MR 89e:14015

[KV] Y. Kawamata and E. Viehweg, On a characterization of abelian varieties in the classification theory of algebraic varieties, Comp. Math. 41 (1981), 355-360. MR 82c:14028

[K] G. Kempf, On the geometry of a theorem of Riemann, Ann. of Math. 98 (1973), 178-185. MR 50:2180

[Kol1] J. Kollár, Higher direct images of dualizing sheaves, I, Ann. of Math 123 (1986), 11-42. MR 87c: 14038

[Kol2] - Shafarevich maps and plurigenera of algebraic varieties, Invent. Math. 113 (1993), 177-215. MR 94m:14018

[Kol3] Shafarevich Maps and Automorphic Forms, Princeton Univ. Press, 1995. MR 96i: 14016

[Kol4] Singularities of pairs (to appear).

[LB] H. Lange and C. Birkenhake, Complex Abelian Varieties, Springer, 1992. MR 94j:14001

[Mori] S. Mori, Classification of higher dimensional varieties, Proc. Symp. Pure Math. 46 (1987), 269-332. MR 89a:14040

[Muk] S. Mukai, Duality between $D(X)$ and $D(\hat{X})$, with application to Picard sheaves, Nagoya Math. J. 81 (1981), 153-175. MR 82f:14036

[Nak] M. Nakamaye, Reducibility of principally polarized abelian varieties with highly singular polarization (to appear).

[SV] R. Smith and R. Varley, Multiplicity g points on theta divisors, Duke Math. Jour. 82 (1996), 319-326. CMP 96:12 
[Var] J. Varouchas, Sur l'image d'une variété Kählérienne compacte, Fonctions de Plusieurs Variables Complexes V, Lecture Notes in Math. 1188 (1986), 245-256. MR 89d:32064

[Z] Qi Zhang, Global holomorphic one-forms and Euler characteristics on projective manifolds with ample canonical bundles (to appear).

Department of Mathematics, University of Illinois at Chicago, Chicago, Illinois 60680

E-mail address: U22425@math.uic.edu

Department of Mathematics, University of California, Los Angeles, California 90024

E-mail address: rkl@math.ucla.edu 